\title{
Global Stability of Nonlinear Stochastic SEI Epidemic Model with Fluctuations in Transmission Rate of Disease
}

\author{
Olusegun Michael Otunuga \\ Department of Mathematics, Marshall University, One John Marshall Drive, Huntington, WV, USA \\ Correspondence should be addressed to Olusegun Michael Otunuga; otunuga@marshall.edu
}

Received 23 October 2016; Accepted 4 January 2017; Published 23 January 2017

Academic Editor: Manuel Osvaldo Cáceres

Copyright (C) 2017 Olusegun Michael Otunuga. This is an open access article distributed under the Creative Commons Attribution License, which permits unrestricted use, distribution, and reproduction in any medium, provided the original work is properly cited.

\begin{abstract}
We derive and analyze the dynamic of a stochastic SEI epidemic model for disease spread. Fluctuations in the transmission rate of the disease bring about stochasticity in model. We discuss the asymptotic stability of the infection-free equilibrium by first deriving the closed form deterministic $\left(R_{0}\right)$ and stochastic $\left(\mathscr{R}_{0}\right)$ basic reproductive number. Contrary to some author's remark that different diffusion rates have no effect on the stability of the disease-free equilibrium, we showed that even if no epidemic invasion occurs with respect to the deterministic version of the SEI model (i.e., $R_{0}<1$ ), epidemic can still grow initially (if $\mathscr{R}_{0}>1$ ) because of the presence of noise in the stochastic version of the model. That is, diffusion rates can have effect on the stability by causing a transient epidemic advance. A threshold criterion for epidemic invasion was derived in the presence of external noise.
\end{abstract}

\section{Introduction}

Many mathematical models have been developed in order to understand disease transmissions and behavior of epidemics. Among these model is the SEI susceptible-exposed-infectious model. This model is used by some author in studying disease transmission of the Severe Acute Respiratory Syndrome (SARS) disease. Several authors [1-3] have studied other models like SEIR and SEIRS to analyze the spread of the disease. Guihua and Zhen [3] studied the deterministic SEI model by providing conditions for the global asymptotic stability of the infection-free and epidemic equilibrium using the higher dimensional Poincare-Bendixson property.

In this paper, we are interested in studying the effect of stochastic fluctuations in the disease transmission rates in the susceptible-exposed-infected epidemic model. We assume that a susceptible individual first goes through latent period after infection before becoming infectious. We consider a case where the disease is infectious in the latent period and the infected period. We study the role of external noise in the transmission rate. We assume the external noise is a Gaussian white noise. According to Méndez et al. [4], Langevin equations that describe system with real noise should be interpreted as a Stratonovich equation, instead of
Ito equation. Due to this reason, we develop a Stratonovich stochastic dynamic SEI model by introducing noise in the transmission rates.

The paper is organized as follows.

In Section 2, we present a Stratonovich stochastic SEI model by allowing the transmission rates to fluctuate around a mean value. The Stratonovich model is now converted into its Ito version. In Section 3, we show that the solution of the stochastic SEI model discussed in Section 2 exists and it is positive. By linearizing the Ito version of the stochastic SEI model around the infection-free equilibrium, we give a closed form expectation of the susceptible, exposed and infected. In Section 4, the closed form value for the stochastic reproductive number $\mathscr{R}_{0}$ is given. This is used to discuss and analyze the stability of the infection-free equilibrium. In Section 5, a numerical simulation is presented to verify our claim. The conclusion of the work is given in Section 6.

\section{Stochastic SEI Model}

We consider the SEI model for description of the population dynamics for SARS and other similar diseases. The host population is partitioned into three compartments: the susceptible, exposed (latent), and infectious, with sizes denoted by $S, E$, 
and $I$, respectively. The total population $N=S+E+I$. The SEI model is described by the following system of differential equation:

$$
\begin{gathered}
d S=\left(-\beta_{1} S E-\beta_{2} S I-\mu S+\delta\right) d t, \quad S\left(t_{0}\right)=S_{0}, \\
d E=\left(\beta_{1} S E+\beta_{2} S I-\gamma E-\left(\mu+\alpha_{1}\right) E\right) d t, \\
E\left(t_{0}\right)=E_{0}, \\
d I=\left(\gamma E-\kappa I-\left(\mu+\alpha_{2}\right) I\right) d t, \quad I\left(t_{0}\right)=I_{0},
\end{gathered}
$$

where $\delta$ is the recruitment constant, $\beta_{1}$ and $\beta_{2}$ are rates of efficient contact in the latent period and infected period, respectively, $\mu>0$ is the natural death rate, $\gamma>0$ is the transfer rate from the exposed to the infectious compartment, $\alpha_{1} \geq 0, \alpha_{2} \geq 0$ are rates of disease-caused death, and $\kappa$ is the rate coefficient of segregation after disease. From (1) and the fact that the total population $N=S+E+I$, we have $N$ satisfying the equation

$$
d N=\left(\delta-\mu N-\alpha_{1} E-\left(\alpha_{2}+\kappa\right) I\right) d t
$$

It follows from (2) that the population size $N$ may vary with time and $\lim _{t \rightarrow \infty} \sup N(t) \leq \delta / \mu$. Hence, we consider model (1) in the feasible region:

$$
T:=\left\{(S, E, I) \in \mathbb{R}_{+}^{3}: 0 \leq S+E+I=N \leq \frac{\delta}{\mu}\right\} .
$$

Here, $\mathbb{R}_{+}$denotes nonnegative real number. It can be shown that $T$ is positively invariant with respect to (1).

The system has two equilibriums: the infection-free equilibrium $P_{0}$ and the endemic equilibrium $P_{1}$. The infectionfree equilibrium $P_{0}:=\left(S^{*}=\delta / \mu, E^{*}=0, I^{*}=0\right)$ exists on the boundary, $\partial T$, of $T$ while the endemic equilibrium $P_{1}=(\mathcal{S}, \mathscr{E}, \mathscr{I})$ exists in the interior of $T$ with

$$
\begin{aligned}
& \mathcal{S}=\frac{\left(\alpha_{2}+\kappa+\mu\right)\left(\alpha_{1}+\mu+\gamma\right)}{\beta_{1}\left(\alpha_{2}+\kappa+\mu\right)+\beta_{2} \gamma}, \\
& \mathscr{E}=\frac{\left(\alpha_{2}+\kappa+\mu\right)\left(\beta_{1} \delta-\mu\left(\alpha_{1}+\gamma+\mu\right)\right)+\beta_{2} \delta \gamma}{\left(\alpha_{1}+\mu+\gamma\right)\left(\beta_{1}\left(\alpha_{2}+\kappa+\mu\right)+\beta_{2} \gamma\right)}, \\
& \mathscr{I}=\frac{\gamma}{\alpha_{2}+\kappa+\mu} \mathscr{E} .
\end{aligned}
$$

By setting $\delta=\mu$, we make the sizes $S, E$, and $I$ into percentages. This reduces the feasible region $T$ to

$$
T:=\left\{(S, E, I) \in \mathbb{R}_{+}^{3}: 0 \leq S+E+I=N \leq 1\right\} .
$$

Let

$$
R_{0}:=\frac{\beta_{1}\left(\kappa+\mu+\alpha_{2}\right)+\beta_{2} \gamma}{\left(\gamma+\mu+\alpha_{1}\right)\left(\kappa+\mu+\alpha_{2}\right)} S^{*} .
$$

If $R_{0} \leq 1, P_{0}$ is the only equilibrium in $T$. If $R_{0}>1$, the unique endemic equilibrium $P_{1}$ exists in $T$. Note that model (1) is similar to the model considered by Guihua and Zhen in [3]. They showed using LaSalle's invariance principle that the disease-free equilibrium, $P_{0}$, is globally asymptotically stable in $T$ if $R_{0} \leq 1$ and unstable if $R_{0}>1$. We define $R_{0}$ in (6) as the deterministic basic reproductive number.

By allowing the transmission rates $\beta_{1}$ and $\beta_{2}$ to fluctuate around a mean value, we introduce external fluctuations in the model as follows:

$$
\beta_{i}(t) \equiv \beta_{i}+\sigma_{i} \mathscr{C}_{i}(t), \quad i=1,2
$$

where $\mathscr{C}_{i}(t), i=1,2$, are independent noise term with zero mean, and $\sigma_{i}>0, i=1,2$ are noise intensity, a measure of the amplitude of fluctuation with respect to $\beta_{i}, i=1,2$, respectively. Substituting (7) into (1), the dynamic model (1) reduces to the Stratonovich stochastic model:

$$
\begin{aligned}
d S= & \left(-\beta_{1} S E-\beta_{2} S I-\mu S+\delta\right) d t-\sigma_{1} S E \circ d W_{1}(t) \\
& -\sigma_{2} S I \circ d W_{2}(t), \quad S\left(t_{0}\right)=S_{0}, \\
d E= & \left(\beta_{1} S E+\beta_{2} S I-\left(\gamma+\mu+\alpha_{1}\right) E\right) d t+\sigma_{1} S E \\
& \circ d W_{1}(t)+\sigma_{2} S I \circ d W_{2}(t), \quad E\left(t_{0}\right)=E_{0} \\
d I= & \left(\gamma E-\left(\kappa+\mu+\alpha_{2}\right) I\right) d t, \quad I\left(t_{0}\right)=I_{0}
\end{aligned}
$$

where $W_{i}(t), i=1,2$, are independent standard Wiener processes defined on stochastic basis $\left(\Omega, \mathscr{F},\left(\mathscr{F}_{t}\right)_{t \geq 0}, \mathbb{P}\right)$; 。 denotes the Stratonovich integral $[4,5]$.

We convert (8) to its Ito's equivalent using the Stratonovich-Ito conversion (Bernardi et al. [6]) given below.

Theorem 1. The Ito Stochastic differential equation

$$
d X=a(t, X) d t+\sum_{j=1}^{M} b^{j}(t, X) d W^{j}(t)
$$

having the same solution as the $N$-dimensional Stratonovich SDE with an M-dimensional Wiener process

$$
d X=\underline{a}(t, X) d t+\sum_{j=1}^{M} b^{j}(t, X) \circ d W^{j}(t)
$$

has drift coefficient $a(t, X)$ that is defined in terms of $\underline{a}(t, X)$, componentwise, by

$$
a^{i}(t, X)=\underline{a}^{i}(t, X)+\frac{1}{2} \sum_{k=1}^{N} \sum_{j=1}^{M} b^{k, j}(t, X) \frac{\partial b^{i, j}}{\partial x_{k}}(t, X) .
$$

Using Theorem 1, the Ito equivalent of (8) is given by

$$
\begin{array}{r}
d S=\left(-\beta_{1} S E-\beta_{2} S I-\mu S+\delta+\frac{1}{2} \sigma_{1}^{2} S E(E-S)\right. \\
\left.+\frac{1}{2} \sigma_{2}^{2} S I^{2}\right) d t-\sigma_{1} S E d W_{1}(t)-\sigma_{2} S I d W_{2}(t), \\
S\left(t_{0}\right)=S_{0},
\end{array}
$$




$$
\begin{aligned}
d E & =\left(\beta_{1} S E+\beta_{2} S I-\left(\gamma+\mu+\alpha_{1}\right) E\right. \\
& \left.-\frac{1}{2} \sigma_{1}^{2} S E(E-S)-\frac{1}{2} \sigma_{2}^{2} S I^{2}\right) d t+\sigma_{1} S E d W_{1}(t) \\
& +\sigma_{2} S I d W_{2}(t), \quad E\left(t_{0}\right)=E_{0}, \\
d I & =\left(\gamma E-\left(\kappa+\mu+\alpha_{2}\right) I\right) d t, \quad I\left(t_{0}\right)=I_{0} .
\end{aligned}
$$

\section{Existence and Uniqueness of Positive Solution of (12)}

In this section, we show that not only does the stochastic model (12) have a unique global solution but also the solution will remain within $T$ whenever it starts from there.

Following Theorem 3.5 of Khasminskii [7], we use Theorem 4 below to show the existence and uniqueness of positive solution of (12).

Definition 2. Let $C_{1,2}(D)$ be the family of nonnegative functions $\mathbf{V}(t, \mathbf{u})$ defined on $D$ such that they are continuously differentiable with respect to $t$ and twice continuously differentiable with respect to $\mathbf{u}$.

Definition 3. Define the domain $U_{n}$ by $U_{n}=\{|x|<n\}$. One defines the differential operator $L$ for a function $\mathbf{V}(t, \mathbf{u}) \in C_{1,2}$ corresponding to a stochastic differential equation with drift and diffusion coefficients $\mathbf{A}(t, \mathbf{u})$ and $\mathbf{B}(t, \mathbf{u})$, respectively, by

$$
\begin{aligned}
L \mathbf{V}(t, \mathbf{u})= & \frac{\partial \mathbf{V}(t, \mathbf{u})}{\partial t}+\mathbf{A}^{T} \frac{\partial \mathbf{V}(t, \mathbf{u})}{\partial \mathbf{u}} \\
& +\frac{1}{2} \operatorname{tr}\left[B^{T} \frac{\partial^{2} \mathbf{V}(t, \mathbf{u})}{\partial \mathbf{u}^{2}} B\right]
\end{aligned}
$$

where $\partial \mathbf{V}(t, \mathbf{u}) / \partial \mathbf{u}=\left(\partial \mathbf{V}(t, \mathbf{u}) / \partial u_{1}, \ldots, \partial \mathbf{V}(t, \mathbf{u}) / \partial u_{n}\right)$ and $\partial^{2} \mathbf{V}(t, \mathbf{u}) / \partial \mathbf{u}^{2}=\left(\partial^{2} \mathbf{V}(t, \mathbf{u}) / \partial u_{i} \partial u_{j}\right)_{i, j}$. Define $\mathfrak{R}_{++}$to be set of positive real numbers. Using the substitution $E=N-$ $S-I$, one reduces (12) to model governing only $S$ and $I$ and applies Theorem 4 to show existence and uniqueness of positive solution of the reduced model.

Theorem 4. Suppose that (12) satisfies the classical existence and uniqueness theorem in every cylinder $[a, b] \times U_{R}$ and, moreover, that there exists a nonnegative function $V \in$ $C_{1,2}\left([0, T] \times \mathfrak{R}_{++}^{2} \rightarrow \mathfrak{R}_{++}\right)$such that for some constant $c>0$

$$
\begin{aligned}
L V & \leq c V \\
V_{R} & =\inf _{|x|>R} V(t, x) \longrightarrow \infty, \text { as } R \longrightarrow \infty .
\end{aligned}
$$

Then, for every random variable $X\left(t_{0}\right)=\left(S\left(t_{0}\right), I\left(t_{0}\right)\right)$ independent of the processes $W(t)-W\left(t_{0}\right)$, there exists a solution $X(t)=(S(t), I(t))$ of the reduced stochastic differential equation (12) (with $E=N-S-I$ ) which is an almost surely continuous stochastic process and is unique up to equivalence.
Proof. It is easy to show that (12) satisfies the classical existence and uniqueness theorem in every cylinder $[a, b] \times$ $U_{R}$. Define $\mathbf{V}:[0, T] \times \Re_{++}^{2} \rightarrow \Re_{++}$by

$$
\mathbf{V}(S, I)=S-\ln (S)+I-\ln (I) .
$$

Note that, for any positive real number $u$, we have $u-\ln (u) \geq 1$ and $u \leq 2(u-\ln (u))-(2-2 \ln (2))$. It then follows that $\mathbf{V} \geq 2$. Also, using the fact that $0 \leq E=N-S-I \leq 1, N \leq 1$, we have

$$
\begin{aligned}
& L \mathbf{V}=\left(1-\frac{1}{S}\right)\left[-\beta_{1} S(N-S-I)-\beta_{2} S I-\mu S+\delta\right. \\
& \left.+\frac{1}{2} \sigma_{1}^{2} S(N-S-I)(N-2 S-I)+\frac{1}{2} \sigma_{2}^{2} S I^{2}\right]+(1 \\
& \left.-\frac{1}{I}\right)\left[\gamma(N-S-I)-\left(\kappa+\mu+\alpha_{2}\right) I\right] \\
& +\frac{1}{2 S^{2}}\left[\sigma_{1}^{2} S^{2}(N-S-I)^{2}+\sigma_{2}^{2} S^{2} I^{2}\right] \leq c V,
\end{aligned}
$$

where $c=\delta+\kappa+2 \mu+\alpha_{2}+2 \max \left\{2 \beta_{1}+2 \gamma, \sigma_{1}^{2} / 2, \sigma_{2}^{2} / 2\right\}$.

It can be easily shown that $\mathbf{V}_{n}=\inf _{|X|>n} \mathbf{V}(t, X) \rightarrow \infty$ as $n \rightarrow \infty$. The result follows. The existence of $E(t)=N-S(t)-$ $I(t)$ follows immediately.

The fact that the solution $(S, E, I)$ of (12) remains in $T$ follows from Corollary 3.1 of Khasminskii [7].

3.1. Closed Form Expectation of Susceptible, Exposed, and Infected Population Near $P_{0}$. We study the condition under which system (12) evolves into an endemic state by analyzing the endemic behavior of the linearized version of (12) around the infection-free equilibrium $P_{0}$.

Using the transformation

$$
\mathbf{u}=\left(\begin{array}{c}
S \\
E \\
I
\end{array}\right)-\left(\begin{array}{c}
S^{*} \\
0 \\
0
\end{array}\right),
$$

we rewrite (12) to get the nonlinear version:

$$
\begin{aligned}
d u_{1}= & f_{1}(t, \mathbf{u}) d t-\left(S^{*}+u_{1}\right) u_{2} \sigma_{1} d W_{1}(t) \\
& -\left(S^{*}+u_{1}\right) u_{3} \sigma_{2} d W_{2}(t), \\
d u_{2}= & f_{2}(t, \mathbf{u}) d t+\left(S^{*}+u_{1}\right) u_{2} \sigma_{1} d W_{1}(t) \\
& +\left(S^{*}+u_{1}\right) u_{3} \sigma_{2} d W_{2}(t), \\
d u_{3}= & f_{3}(t, \mathbf{u}) d t
\end{aligned}
$$

where

$$
\begin{aligned}
f_{1}(t, \mathbf{u})= & -\beta_{1}\left(S^{*}+u_{1}\right) u_{2}-\beta_{2}\left(S^{*}+u_{1}\right) u_{3}-\mu u_{1} \\
& -\frac{1}{2}\left(S^{*}+u_{1}\right)^{2} u_{2} \sigma_{1}^{2}+\frac{1}{2}\left(S^{*}+u_{1}\right) u_{2}^{2} \sigma_{1}^{2} \\
& +\frac{1}{2}\left(S^{*}+u_{1}\right) u_{3}^{2} \sigma_{2}^{2},
\end{aligned}
$$




$$
\begin{aligned}
f_{2}(t, \mathbf{u})= & \beta_{1}\left(S^{*}+u_{1}\right) u_{2}+\beta_{2}\left(S^{*}+u_{1}\right) u_{3} \\
& -\left(\gamma+\mu+\alpha_{1}\right) u_{2}+\frac{1}{2}\left(S^{*}+u_{1}\right)^{2} u_{2} \sigma_{1}^{2} \\
& -\frac{1}{2}\left(S^{*}+u_{1}\right) u_{2}^{2} \sigma_{1}^{2}-\frac{1}{2}\left(S^{*}+u_{1}\right) u_{3}^{2} \sigma_{2}^{2}, \\
f_{3}(t, \mathbf{u})= & \gamma u_{2}-\left(\kappa+\mu+\alpha_{2}\right) u_{3} .
\end{aligned}
$$

The linearization of (12) around the infection-free equilibrium $P_{0}$ is equivalent to the linearization of (18) around its trivial solution $(\mathbf{u}=0)$, given by

$$
\begin{aligned}
d \mathbf{u}=\mathbf{A} \mathbf{u} d t+\mathbf{B}_{1} \mathbf{u} d W_{1}(t)+\mathbf{B}_{2} \mathbf{u} d W_{2}(t), & \\
& \mathbf{u}\left(t_{0}\right)=\mathbf{u}_{0},
\end{aligned}
$$

where

$$
\begin{aligned}
\mathbf{A} & =\left(\begin{array}{ccc}
-\mu & -\left(\beta_{1}+\frac{\sigma_{1}^{2} S^{*}}{2}\right) S^{*} & -\beta_{2} S^{*} \\
0 & -\nu & \beta_{2} S^{*} \\
0 & \gamma & -\left(\kappa+\mu+\alpha_{2}\right)
\end{array}\right), \\
\mathbf{B}_{1} & =\left(\begin{array}{ccc}
0 & -\sigma_{1} & 0 \\
0 & \sigma_{1} & 0 \\
0 & 0 & 0
\end{array}\right) S^{*}, \\
\mathbf{B}_{2} & =\left(\begin{array}{ccc}
0 & 0 & -\sigma_{2} \\
0 & 0 & \sigma_{2} \\
0 & 0 & 0
\end{array}\right) S^{*}, \\
\nu & =\gamma+\mu+\alpha_{1}-\left(\beta_{1}+\frac{\sigma_{1}^{2} S^{*}}{2}\right) S^{*} .
\end{aligned}
$$

It follows that the expected value, $\mathbb{E}[\mathbf{u}(t)]$, of the solution of (20) is given by

$$
\mathbb{E}[\mathbf{u}(t)]=\left(\begin{array}{c}
m_{1} e^{-z_{1} t}+c_{1} e^{-z_{2} t}+c_{2} e^{-z_{3} t} \\
\bar{m}_{2} e^{-z_{2} t}+\bar{m}_{3} e^{-z_{3} t} \\
m_{2} e^{-z_{2} t}+m_{3} e^{-z_{3} t}
\end{array}\right)
$$

where $m_{i}=\mathbb{E}\left[u_{i}\left(t_{0}\right)\right], i=1,2,3, \bar{m}_{i}=\left(m_{i} / \gamma\right)\left(\kappa+\mu+\alpha_{2}-z_{i}\right)$, $c_{1}=\left(\left(\beta_{2} m_{2}+\left(\beta_{1}+\sigma_{1}^{2} S^{*} / 2\right) \bar{m}_{2}\right) /\left(z_{2}-\mu\right)\right) S^{*}, c_{2}=\left(\left(\beta_{2} m_{3}+\right.\right.$ $\left.\left.\left(\beta_{1}+\sigma_{1}^{2} S^{*} / 2\right) \bar{m}_{3}\right) /\left(z_{3}-\mu\right)\right) S^{*}, z_{1}=\mu, z_{i}=(1 / 2)\left[\left(\alpha_{2}+\kappa+\right.\right.$ $\left.\mu+\nu)+(-1)^{i} \sqrt{\left(\alpha_{2}+\kappa+\mu-\nu\right)^{2}+4 \gamma \beta_{2} S^{*}}\right], i=2,3$.

\section{Stability Analysis of Infection-Free Equilibrium $P_{0}$}

Using Theorem A.2 of Tornatore et al. [2], we show the global stability of the nonlinear stochastic system (12). Notice that, in order to avoid epidemic invasion, we must have $z_{i}>0$, $i=1,2,3$. This is equivalent to

$$
\begin{gathered}
\left(\beta_{1}+\frac{\sigma_{1}^{2}}{2} S^{*}\right)\left(\kappa+\mu+\alpha_{2}\right) S^{*}+\beta_{2} \gamma S^{*} \\
-\left(\gamma+\mu+\alpha_{1}\right)\left(\kappa+\mu+\alpha_{2}\right)<0 .
\end{gathered}
$$

Also (23) implies that $v>0$.

Define

$$
\mathscr{R}_{0}=\frac{\left(\beta_{1}+\left(\sigma_{1}^{2} / 2\right) S^{*}\right)\left(\kappa+\mu+\alpha_{2}\right)+\beta_{2} \gamma}{\left(\gamma+\mu+\alpha_{1}\right)\left(\kappa+\mu+\alpha_{2}\right)} S^{*} \text {. }
$$

Remark 5. It follows that (23) is equivalent to $\mathscr{R}_{0}<1$. We can rewrite $\mathscr{R}_{0}$ in terms of $R_{0}$ by combining (6) and (24) as follows:

$$
\mathscr{R}_{0}=R_{0}+\frac{\sigma_{1}^{2}\left(S^{*}\right)^{2}}{2\left(\gamma+\mu+\alpha_{1}\right)} .
$$

We call the constant $\mathscr{R}_{0}$ defined in (25) the stochastic basic reproductive number.

Using the following theorem, we get conditions for stochastic asymptotic stability of the infection-free equilibrium.

Definition 6. The solution $\mathbf{u}(t)=0$ of system (20) is said to be

(1) $p$-stable $(p>0)$ for $t \geq 0$, if

$$
\sup _{|\mathbf{u}| \leq n, t \geq s} \mathbb{E}|\mathbf{u}(t)|^{p} \longrightarrow 0, \quad \text { as } n \longrightarrow 0,(s \geq 0) ;
$$

(2) asymptotically $p$-stable, if it is $p$-stable and moreover $\mathbb{E}|\mathbf{u}(t)|^{p} \rightarrow 0$ as $t \rightarrow \infty ;$

(3) exponentially $p$-stable, if, for some positive constants $A$ and $c$,

$$
\mathbb{E}|\mathbf{u}(t)|^{p} \leq A|\mathbf{u}|^{p} \exp [-c(t-s)] ;
$$

(4) when $p=1$, one says stability in the mean and for $p=2$, one says stability in mean square.

Theorem 7. If

$$
\mathscr{R}_{0}<1
$$

holds, then the solution $\mathbf{u}(t)=0$ of (20) is globally asymptotically stable. Furthermore, if $R_{0}>1$, the solution $\mathbf{u}(t)=0$ is unstable.

Proof. If (28) holds, it follows from (25) that $R_{0}<1$ and (22) implies $\lim _{t \rightarrow \infty} \mathbb{E}[|\mathbf{u}(t)|]=0$. If $R_{0}>1$, then $\mathscr{R}_{0}>1$ and $\mathbb{E}[|\mathbf{u}(t)|] \rightarrow \infty$ as $t \rightarrow \infty$.

Remark 8. Contrary to Remark 3.1 of Kim and Lin [1], if

$$
1-\frac{\sigma_{1}^{2}\left(S^{*}\right)^{2}}{2\left(\gamma+\mu+\alpha_{1}\right)}<R_{0}<1,
$$




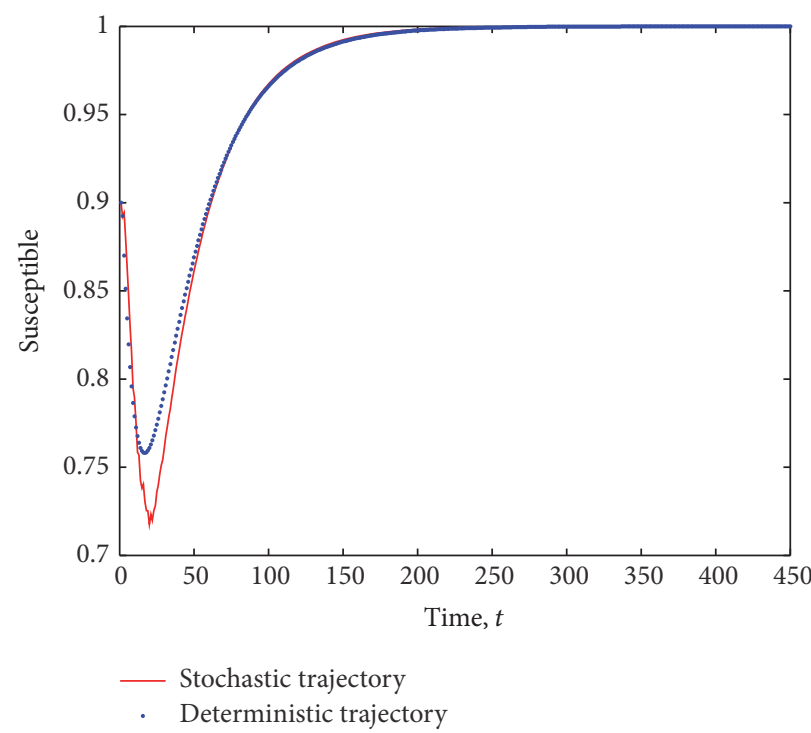

(a)

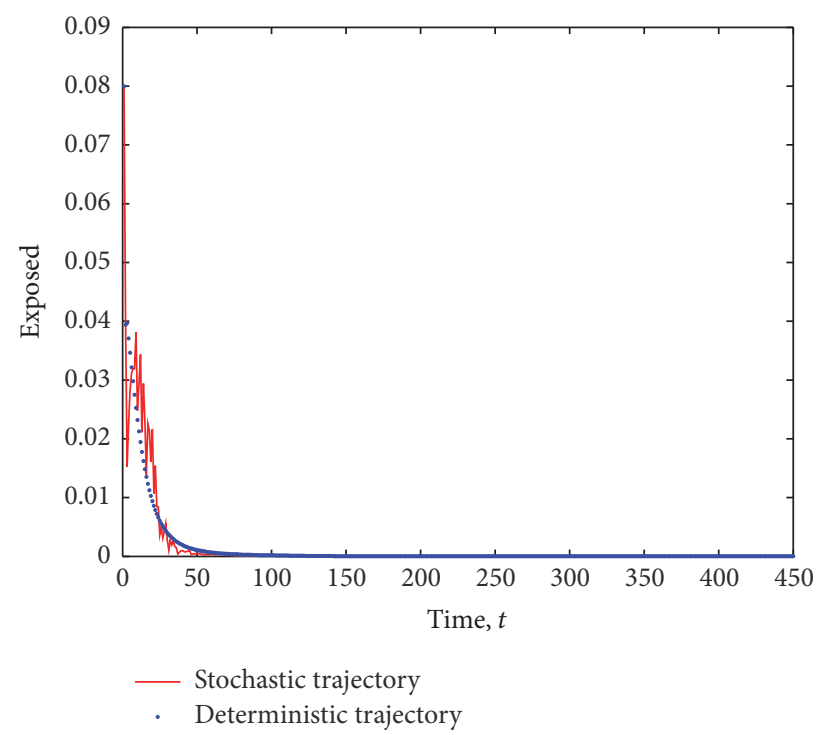

(b)

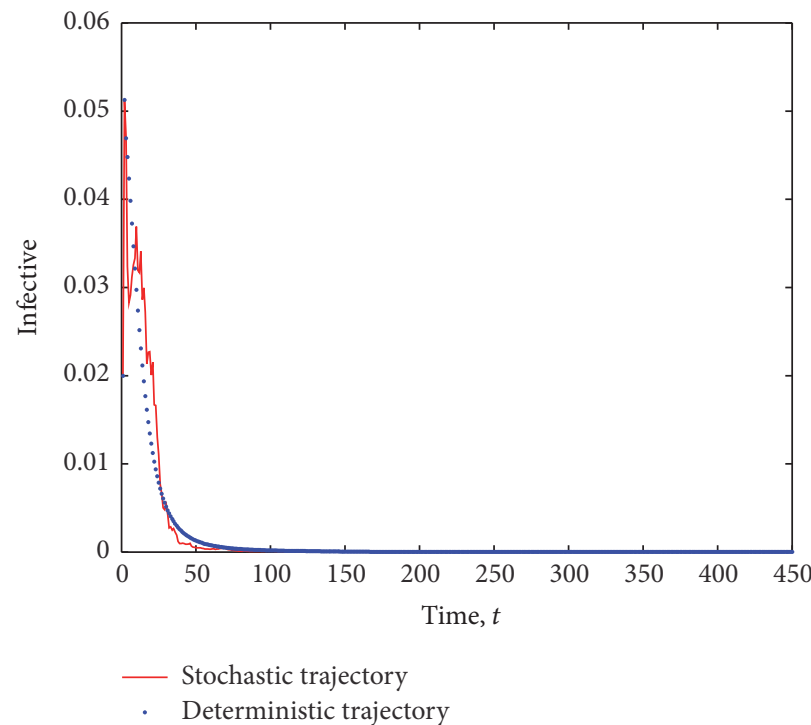

(c)

FIGURE 1: Graph of deterministic and stochastic trajectories of SEI model for initial conditions $S_{0}=0.9, E_{0}=0.08, I_{0}=0.02, \sigma_{1}=0.15$, $\sigma_{2}=0.8, R_{0}=0.9346$, and $\mathscr{R}_{0}=0.9845 ;\left(R_{0}<1, \mathscr{R}_{0}<1\right)$.

then epidemic can grow initially, leading to transient epidemic advance. Note that condition (29) implies $R_{0}<1$ and $\mathscr{R}_{0}=R_{0}+\sigma_{1}^{2}\left(S^{*}\right)^{2} / 2\left(\gamma+\mu+\alpha_{1}\right)>1$. Hence, $z_{1}>0, z_{2}>0$, and $z_{3}<0$. The transient epidemic advance is caused by the noise intensity, $\sigma_{1}$, in the rate of efficient contact in the latent period, $\beta_{1}$.

Remark 9. Note that the global stability of the trivial solution $\mathbf{u}=0$ of (20) is equivalent to the global stability of the infection-free equilibrium $P_{0}=\left(S^{*}, 0,0\right)$.

The following theorem shows that the expected value $\mathbb{E}[\mathbf{u}(t)]$ in (22) does not always converge to the trivial solution $\mathbf{u}=0$ of (20) if $\mathscr{R}_{0}=1$. In fact, if $\mathscr{R}_{0}=1$, then $\mathbb{E}[\mathbf{u}(t)] \rightarrow 0$ as $t \rightarrow \infty$ if $m_{3}=\mathbb{E}\left[u_{3}\left(t_{0}\right)\right]=\mathbb{E}\left[I\left(t_{0}\right)\right]=0$.

Theorem 10. If $\mathscr{R}_{0}=1$, then $\mathbb{E}[\mathbf{u}(t)]$ in (22) converge to the trivial solution $\mathbf{u}=0$ of (20) if $\mathbb{E}\left[u_{3}\left(t_{0}\right)\right]=0$.

Proof. From (23) and (24), $\mathscr{R}_{0}=1$ is equivalent to $\left[\left(\beta_{1}+\right.\right.$ $\left.\left.\left(\sigma_{1}^{2} / 2\right) S^{*}\right)\left(\kappa+\mu+\alpha_{2}\right)+\beta_{2} \gamma\right] S^{*}=\left(\gamma+\mu+\alpha_{1}\right)\left(\kappa+\mu+\alpha_{2}\right)$. If $\mathscr{R}_{0}=1$, then $v=\left(\beta_{2} \gamma /\left(\kappa+\mu+\alpha_{2}\right)\right) S^{*}, z_{1}=\mu, z_{2}=$ $\alpha_{2}+\kappa+\mu+\nu, z_{3}=0$, and $\mathbb{E}[\mathbf{u}(t)] \rightarrow\left(\begin{array}{c}\frac{\mathcal{C}_{2}}{m_{3}} \\ m_{3}\end{array}\right)$ as $t \rightarrow \infty$, where $c_{2}=-\left(\left(\gamma+\mu+\alpha_{1}\right)\left(\kappa+\mu+\alpha_{2}\right) / \gamma \mu\right) m_{3} S^{*}, \bar{m}_{3}=\left(\left(\kappa+\mu+\alpha_{2}\right) / \gamma\right) m_{3}$. If $m_{3}=0$, then $\mathbb{E}[\mathbf{u}(t)] \rightarrow 0$ as $t \rightarrow \infty$. 


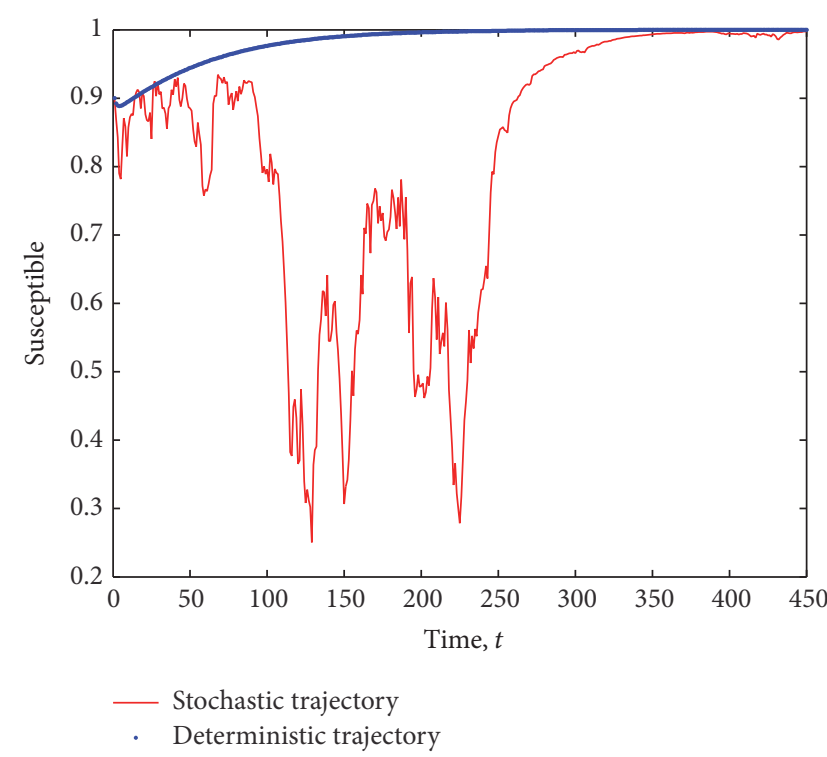

(a)

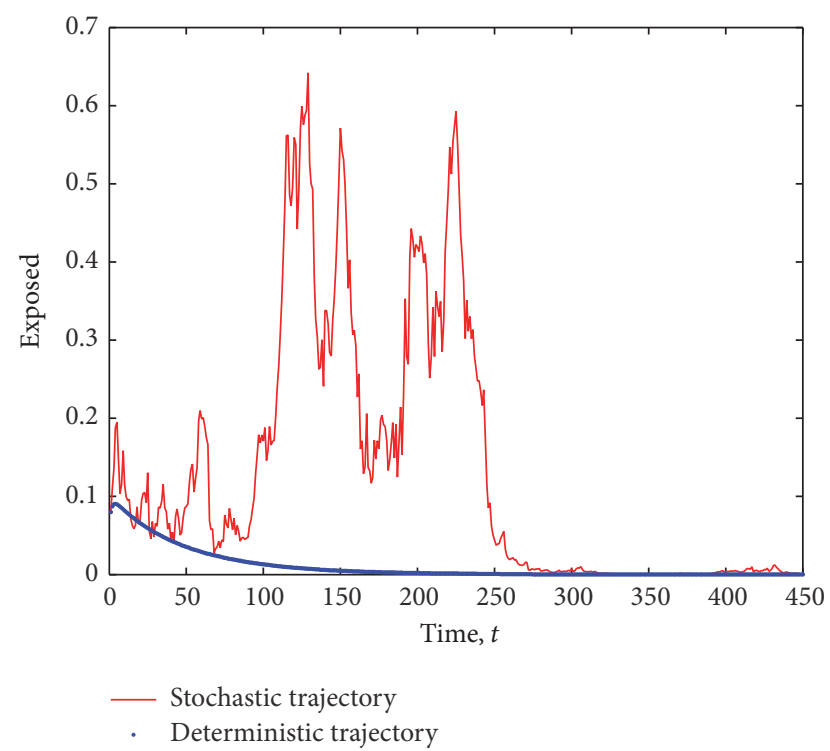

(b)

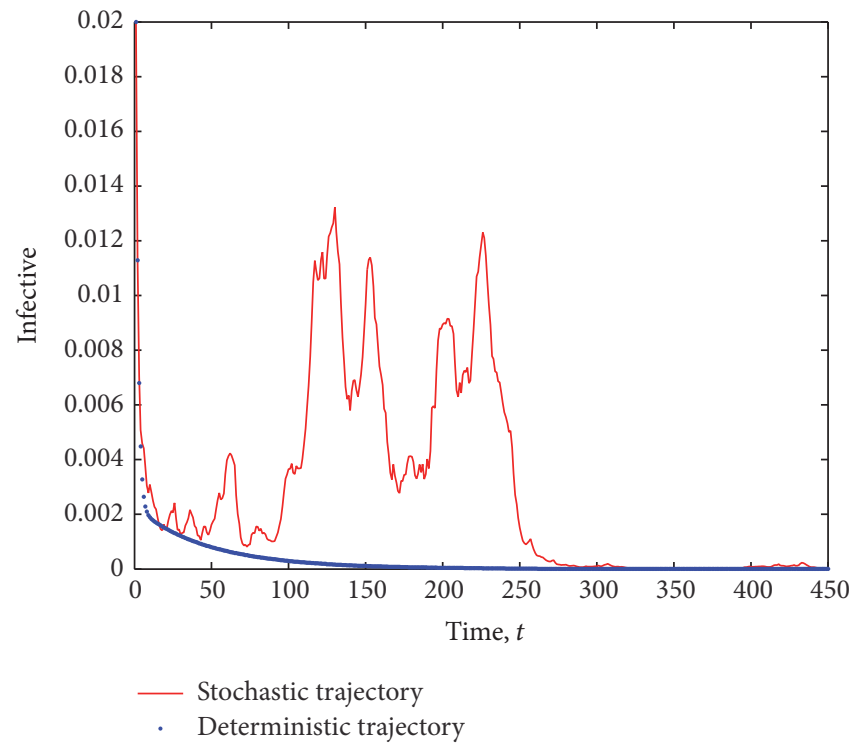

(c)

FIGURE 2: Graph of deterministic and stochastic trajectories of SEI model for the case $1-\sigma_{1}\left(S^{*}\right)^{2} / 2\left(\gamma+\mu+\alpha_{1}\right)<R_{0}<1$ of Remark 8 with initial conditions $S_{0}=0.9, E_{0}=0.08, I_{0}=0.02, \beta_{1}=0.1, \beta_{2}=5.5, \mu=0.3=\delta\left(S^{*}=1\right), \gamma=0.103, \kappa=3.47, \alpha_{1}=0.01, \alpha_{2}=1, \sigma_{1}=0.9$, $\sigma_{2}=0.1, R_{0}=0.5297$, and $\mathscr{R}_{0}=1.5103$.

We show the global stability of the infection-free equilibrium $P_{0}$ and trivial solution $\mathbf{u}=0$ of the nonlinear stochastic systems (12) and (18), respectively.

Theorem 11. If the trivial solution $\mathbf{u}(t)=0$ for linear system (20) with drift and diffusion coefficients $F(t, \mathbf{u})$ and $G(t, \mathbf{u})$, respectively, is asymptotically stable and the drift and diffusion coefficients $f(t, \mathbf{u})$ and $g(t, \mathbf{u})$, respectively, of nonlinear system (18) satisfy the inequality

$$
|f(t, \mathbf{u})-F(t, \mathbf{u})|+|g(t, \mathbf{u})-G(t, \mathbf{u})|<c|\mathbf{u}|
$$

in a sufficiently small neighbourhood of $\mathbf{u}=0$, with $a$ sufficiently small constant $c$, then the trivial solution $\mathbf{u}(t)=0$ of system (18) is globally asymptotically stable.

Proof. In a sufficiently small neighbourhood of $\mathbf{u}=0$, choose $\epsilon>0$ sufficiently small so that $|\mathbf{u}|<\epsilon$. We have

$$
\begin{aligned}
& |f(t, \mathbf{u})-F(t, \mathbf{u})|+|g(t, \mathbf{u})-G(t, \mathbf{u})| \\
& \quad=\left[\left(-\beta_{1} u_{1} u_{2}-\beta_{2} u_{1} u_{3}-\frac{1}{2}\left(S^{*}+u_{1}\right)^{2} u_{2} \sigma_{1}^{2}\right.\right.
\end{aligned}
$$




$$
\begin{aligned}
& +\frac{1}{2}\left(S^{*}+u_{1}\right) u_{2}^{2} \sigma_{1}^{2}+\frac{1}{2}\left(S^{*}+u_{1}\right) u_{3}^{2} \sigma_{2}^{2} \\
& \left.+\frac{\sigma_{1}^{2}\left(S^{*}\right)^{2} u_{2}}{2}\right)^{2}+\left(\beta_{1} u_{1} u_{2}+\beta_{2} u_{1} u_{3}\right. \\
& +\frac{1}{2}\left(S^{*}+u_{1}\right)^{2} u_{2} \sigma_{1}^{2}-\frac{1}{2}\left(S^{*}+u_{1}\right) u_{2}^{2} \sigma_{1}^{2} \\
& \left.\left.-\frac{1}{2}\left(S^{*}+u_{1}\right) u_{3}^{2} \sigma_{2}^{2}-\frac{\sigma_{1}^{2}\left(S^{*}\right)^{2} u_{2}}{2}\right)^{2}\right]^{1 / 2} \\
& +\left[2\left(u_{1} u_{2} \sigma_{1}\right)^{2}+2\left(u_{1} u_{3} \sigma_{2}\right)^{2}\right]^{1 / 2} \leq\left(2 \beta_{1}+2 \beta_{2}\right. \\
& \left.+\sigma_{1}^{2}+\sigma_{2}^{2}+\sqrt{2}\left(\sigma_{1}+\sigma_{2}\right)\right) \epsilon\left|u_{1}\right|+\left(3 S^{*}+1\right) \\
& \cdot \sigma_{1}^{2} \epsilon\left|u_{2}\right|+\sigma_{2}^{2} S^{*} \epsilon\left|u_{3}\right|<c|\mathbf{u}|,
\end{aligned}
$$

where $c=\left(2 \beta_{1}+2 \beta_{2}+\left(3 S^{*}+2\right) \sigma_{1}^{2}+\left(S^{*}+1\right) \sigma_{2}^{2}+\sqrt{2}\left(\sigma_{1}+\right.\right.$ $\left.\left.\sigma_{2}\right)\right) \epsilon$

\section{Numerical Simulations}

Figure 1 verifies the global stability criteria discussed in Theorems 7, 10, and 11 and Remark 8.

Figure 1 shows the graph of deterministic and stochastic trajectories of SEI model for initial conditions $S_{0}=0.9, E_{0}=$ 0.08 , and $I_{0}=0.02$. Here, we see that both $R_{0}$ and $\mathscr{R}_{0}$ are both less than one and both trajectories converge to the infectionfree equilibrium $\left(S^{*}, 0,0\right)=(1,0,0)$.

The transient epidemic advance is evident in all Figures 2(a), 2(b), and 2(c). The number of exposed (latent) individuals drastically increased for some time period. During the same period, the number of infected individuals increased and the number of susceptible individuals decreased.

\section{Conclusion}

The purpose of this work is to shed more light on the effects of the presence of external perturbations (in the form of white noise) in the disease transmission rates in a SEI model. By extending a SEI system of deterministic differential equations into a system of stochastic differential equations, we show the existence of solution of the stochastic model and derive the stochastic basic reproductive number, $\mathscr{R}_{0}$, corresponding to the deterministic reproductive number, $R_{0}$. The stochastic basic reproductive number $\mathscr{R}_{0}$ converges to the deterministic basic reproductive number $R_{0}$ as the noise intensity $\sigma_{1}$ tends to zero. We show that even if the infection-free equilibrium is stable, we might still have a transient epidemic advance. A condition in which this advance can happen is derived and analyzed. From our result, we conclude that the transient epidemic advance is induced by the noise intensity, $\sigma_{1}$, of the white noise. It follows from (7) that as the noise intensity $\sigma_{1}$ increases, the fluctuations around the mean number of infected individuals also increase thereby causing an initial epidemic growth provided that (29) is satisfied.

\section{Competing Interests}

The author declares that there is no conflict of interests regarding the publication of this paper.

\section{References}

[1] K. I. Kim and Z. Lin, "Asymptotic behavior of an SEI epidemic model with diffusion," Mathematical and Computer Modelling, vol. 47, no. 11-12, pp. 1314-1322, 2008.

[2] E. Tornatore, S. M. Buccellato, and P. Vetro, "Stability of a stochastic SIR system," Physica A, vol. 354, no. 1-4, pp. 111-126, 2005.

[3] L. Guihua and J. Zhen, "Global stability of an SEI epidemic model," Chaos, Solitons \& Fractals, vol. 21, no. 4, pp. 925-931, 2004.

[4] V. Méndez, D. Campos, and W. Horsthemke, "Stochastic fluctuations of the transmission rate in the susceptible-infectedsusceptible epidemic model," Physical Review E, vol. 86, no. 1, Article ID 011919, 8 pages, 2012.

[5] L. Arnold, Stochastic Differential Equations: Theory and Applications, John Wiley \& Sons, New York, NY, USA, 1974.

[6] C. Bernardi, Y. Madday, J. F. Blowey, J. P. Coleman, and A. W. Craig, Theory and Numerics of Differential Equations, Springer, Berlin, Germany, 2001.

[7] R. Khasminskii, Stochastic stability of differential equations, vol. 66 of Stochastic Modelling and Applied Probability, Springer Berlin Heidelberg, second edition, 2012. 


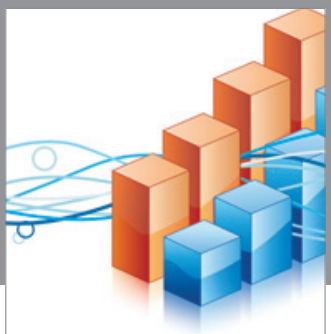

Advances in

Operations Research

vatem alat4

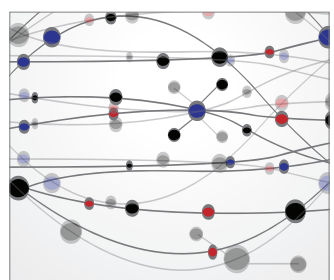

\section{The Scientific} World Journal
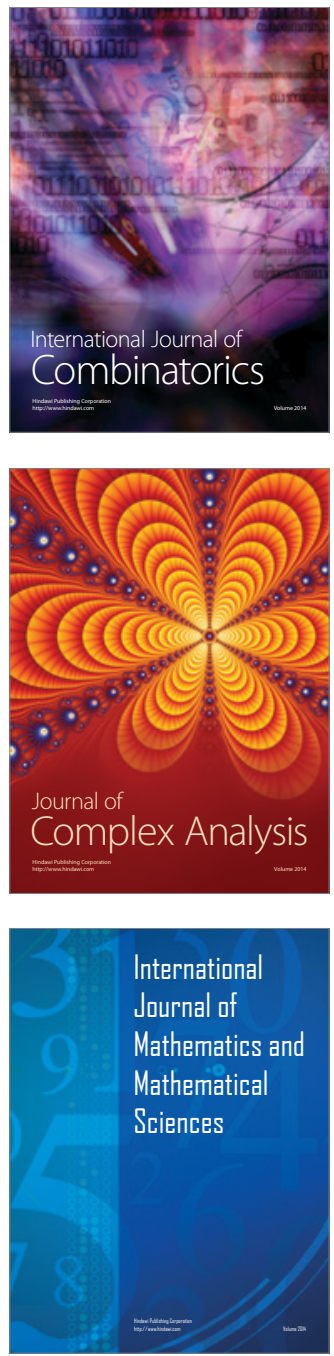
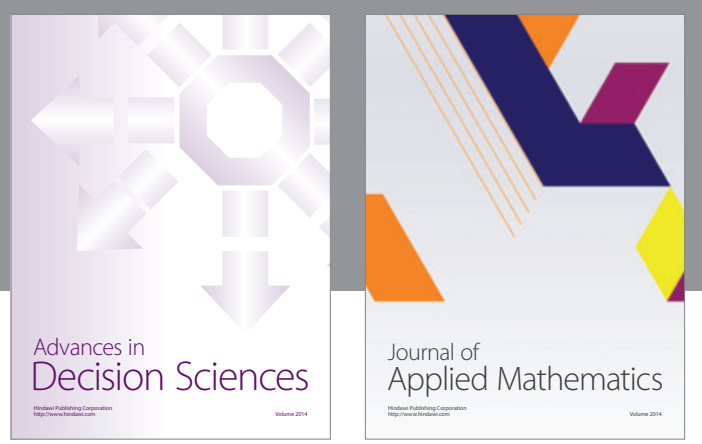

Algebra

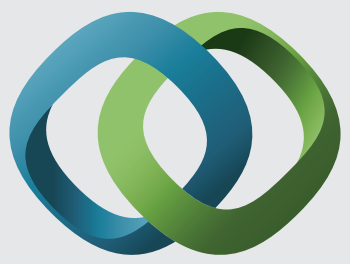

\section{Hindawi}

Submit your manuscripts at

https://www.hindawi.com
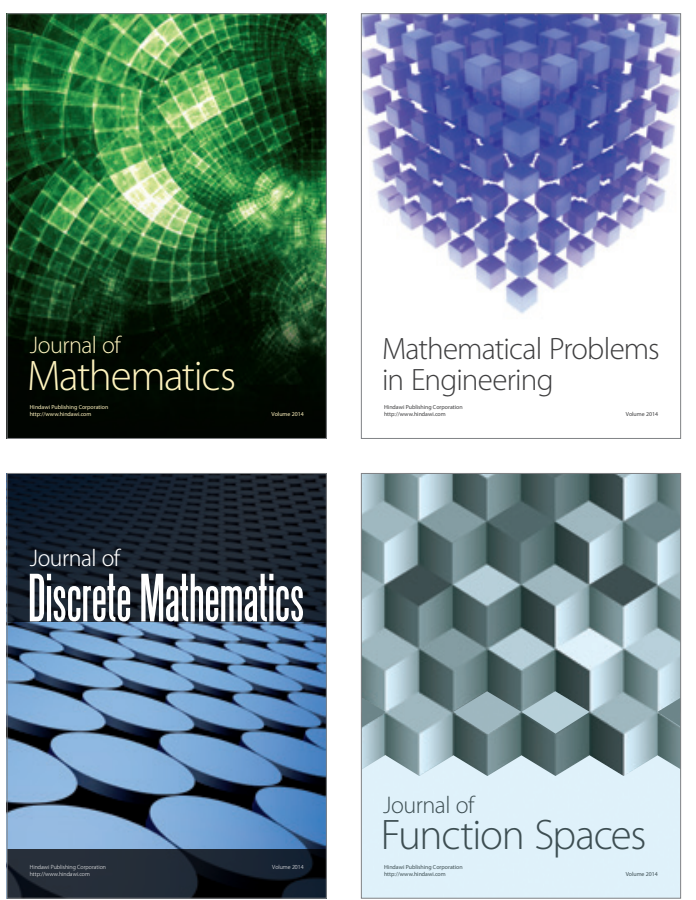

Mathematical Problems in Engineering
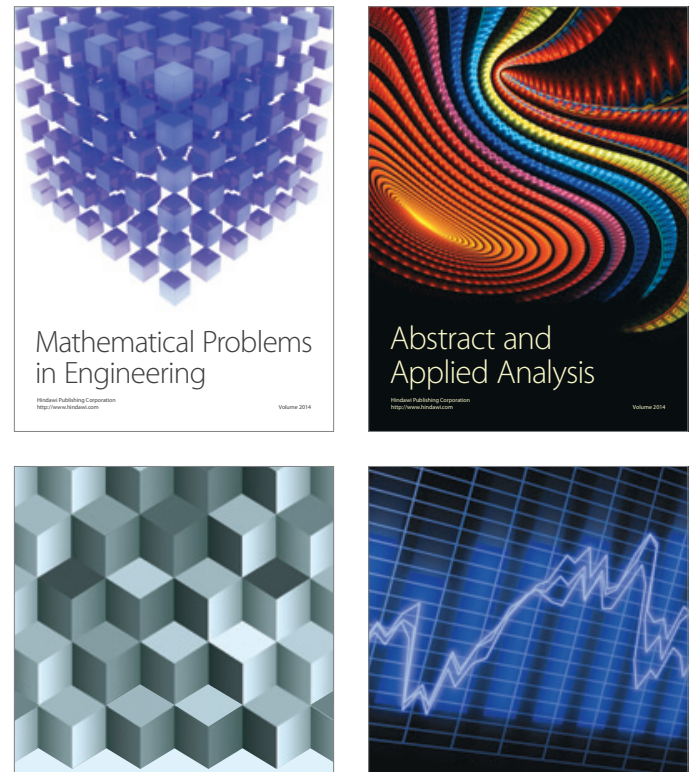

Journal of

Function Spaces

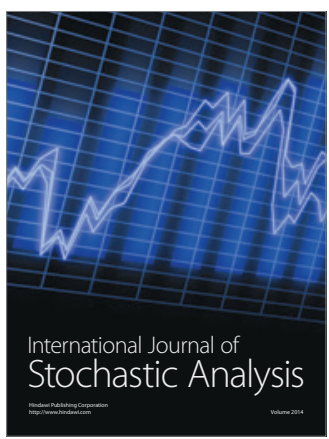

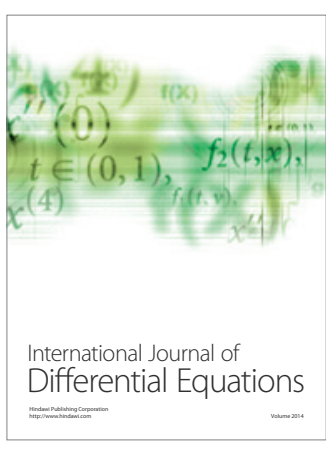
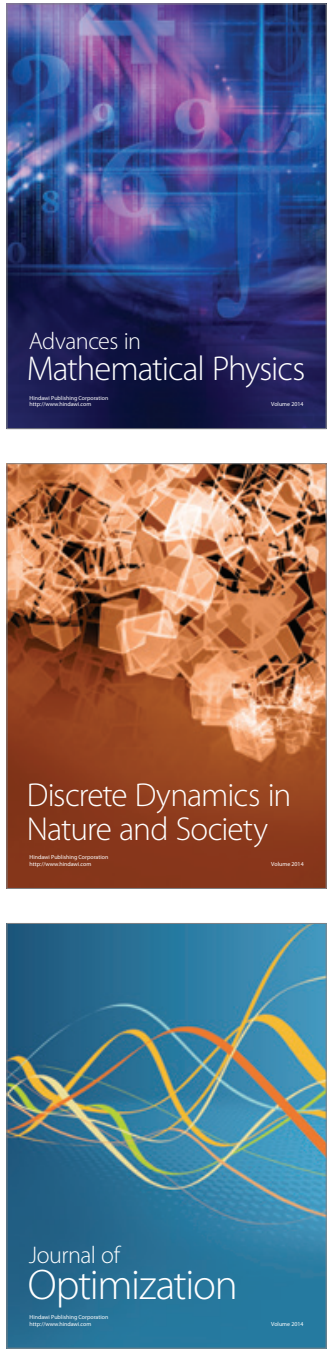